\title{
Contribution of REM sleep and N2 sleep spindles to procedural memory consolidation in Vipassana meditators and non-meditating controls.
}

Elizaveta Solomonova $^{\mathrm{a}, \mathrm{b}}$, Simon Dubé ${ }^{\mathrm{a}, \mathrm{c}}$, Cloé Blanchette-Carrière ${ }^{\mathrm{a}, \mathrm{b}}$, Arnaud Samson-Richer ${ }^{\mathrm{a}, \mathrm{c}}$, Michelle Carr ${ }^{\mathrm{a}, \mathrm{b}}$, Tyna Paquette ${ }^{\mathrm{a}}$ and Tore Nielsen ${ }^{\mathrm{a}, \mathrm{d} *}$

${ }^{a}$ Dream and Nightmare Laboratory, Centre for Advanced Research in Sleep Medicine, CIUSSSNÎM - HSCM, Montréal, Québec, Canada

${ }^{\mathrm{b}}$ Biomedical Sciences Department, Université de Montréal, Montréal, Québec, Canada

${ }^{\mathrm{c}}$ Psychology Department, Université de Montréal, Montréal, Québec, Canada

${ }^{\text {d }}$ Psychiatry Department, Université de Montréal, Montréal, Québec, Canada

*Corresponding author: Tore Nielsen, Center for Advanced Research in Sleep Medicine, CIUSSSNÎM - Hôpital du Sacré-Coeur de Montréal, 5400 Gouin Blvd West, Montreal, Quebec, H4J 1C5, Canada. tore.nielsen@umontreal.ca

\begin{abstract}
Study objectives Rapid eye movement (REM) sleep, non-rapid eye movement (NREM) sleep, and sleep spindles are all implicated in the consolidation of procedural memories. The relative contributions of sleep stages and sleep spindles was previously shown to depend on individual differences in task processing. Experience with Vipassana meditation is one such individual difference that has not been investigated in relation to sleep. Vipassana meditation is a form of mental training that enhances proprioceptive and somatic awareness and alters attentional style. The
\end{abstract}


goal was thus to examine a potential moderating role for Vipassana meditation experience on sleepdependent procedural memory consolidation.

Methods Groups of Vipassana meditation practitioners $(\mathrm{N}=20)$ and matched meditation-naïve controls $(\mathrm{N}=20)$ slept for a single daytime nap in the laboratory. Before and after the nap they completed a procedural task on the Wii Fit balance platform.

Results Meditators performed slightly better on the task before the nap, but the two groups improved similarly after sleep. The groups showed different patterns of sleep-dependent procedural memory consolidation: in meditators task learning was negatively correlated with density of fast and positively correlated with density of slow occipital spindles, while in controls task improvement was associated with increases in REM sleep. Meditation practitioners had a lower density of sleep spindles, especially in occipital regions.

Conclusions Results suggest that neuroplastic changes associated with sustained meditation practice may alter overall sleep architecture and reorganize sleep-dependent patterns of memory consolidation. The lower density of spindles in meditators may mean that meditation practice compensates for some of the memory functions of sleep.

Keywords: Procedural memory; Vipassana meditation; REM sleep; sleep spindles; body awareness

\section{Introduction}

Recent cognitive science and philosophy of mind theories have seen an important shift of focus from inquiry focused predominantly on the role of the brain in subjective experience to the view that the brain is a part of a whole body and is intimately rooted in the physical and social world [14]. From this perspective, much current research focuses on relationships between awareness of one's body and physical and mental health. Mindfulness interventions, including sitting meditation 
practices and movement-based practices such as yoga or t'ai chi [5], all include an element of cultivating access to and awareness of increasingly subtle bodily sensations [6, 7]. Meditation practitioners, therefore, may represent an 'expert' group of healthy individuals who intentionally cultivate attention to bodily states, and are better able to access and employ them [8]. Meditators may embody cognitive processes differently than do non-meditators; in particular, they may rely more upon awareness of kinesthetic and other types of endogenous information to encode memories. The term body awareness captures this characteristic, referring to an ability to focus on, and become familiar with, one's bodily sensations; it is defined as a subjective, explicitly available aspect of proprioception and interoception [9]. Critically, while some individuals may be naturally more attuned to body awareness than others, it is a trainable skill that can be enhanced by contemplative practices $[10,11]$.

The main research goal of the current study is to investigate whether meditation practice, by virtue of increasing body awareness, also changes the neurobiological and phenomenological qualities of sleep-dependent memory consolidation. Specifically, we chose a full body procedural memory task and a daytime nap protocol to test whether: 1) meditation practitioners and meditationnaïve controls differ in performance and improvement on a procedural balance task; 2) the two groups differ in their patterns of sleep stage and sleep microarchitecture in reaction to the task; and 3) daytime nap sleep characteristics (sleep stages and sleep spindles) in meditation practitioners are similar in quality to nighttime sleep.

\section{Memory and sleep}

Traditionally, human long-term memory has been divided into the two broad categories of explicit/declarative and implicit/non-declarative memory $[12,13]$. Explicit memory deals with autobiographical events, is characterized by self-awareness and autonoetic consciousness or the ability to "mentally time travel" through past experience, and is responsible for accumulating semantic knowledge, or facts about the world [14]. Crucially, explicit memory is accessible to 
conscious retrieval [15]. Implicit memory, on the other hand, concerns procedural knowledge, the learning of skills, behavioral conditioning and priming effects and is largely inaccessible to conscious report [16].

Consolidation of procedural memory in sleep has been studied using a number of tasks, including sequential finger tapping [17-24]; serial-reaction time task [25-27]; motor sequence task $[28,29]$; mirror-tracing task [30-33]; button-box sequence [34]; visuomotor adaptation task [23]; texture discrimination task $[35,36]$; visual discrimination task [37], and others. These tasks usually involve either fine motor skills using the fingers or hand-eye coordination, but do not typically involve balance or bodily displacements.

Tasks involving balance skills or full-body learning have only rarely been used in memory consolidation research [38] [39, 40]. One example specific to sleep research is the Alpine Racer arcade game used in a study of memory incorporation into dream content [41].

\section{Sleep and memory consolidation}

A wealth of research supports a role for sleep in memory consolidation [for reviews see 42, 43, 44]. Sleep is thought to strengthen information learned during the day, to select which experiences are best remembered and which best forgotten, and to assimilate new knowledge into existing autobiographical networks [45-48]. While most research has focused on the effects of a full night of sleep, an increasing number of studies report that daytime naps have similar effects to nighttime sleep on memory processes [49-56].

A number of studies link procedural memory to rapid-eye-movement (REM) sleep processes; these include a variety of tasks: mirror-tracing [57], Corsi block tapping, Tower of Hanoi [33, 58] and others. For example, among obstructive sleep apnea patients, reduced rate of REM eye movements (REM density) was associated with poor mirror-tracing performance [59]. Similarly, selective REM sleep deprivation disrupted visual discrimination learning [60]. Yet other studies suggest an important role for NREM sleep in procedural memory consolidation. NREM sleep 
duration [61] as well as NREM electrophysiological events, such as sleep spindles [62, 63], have been associated with post-sleep task improvement.

Sleep's role in memory consolidation is not only influenced by task type and sleep stage/sleep microarchitecture, but also by individual differences in learning abilities and cognitive styles. In one study, for example [64], the ability to bring a motor task into explicit awareness improved postsleep performance on the task, suggesting that training a particular attentional engagement with the learning task may change the neurocognitive style of offline memory processing.

In sum, mounting evidence now links both REM and NREM sleep to procedural memory consolidation. In fact, the same procedural task has been shown to be related, in different contexts, to both REM and NREM sleep. While it is not yet clear why task performance may be associated with one or the other sleep stage — or both — some authors have proposed 2-stage or sleep organization models to account for the findings, i.e., that both stages are necessary for successful consolidation and integration of newly encoded information $[65,66]$, including procedural skills [67]. However, such models do not explain how the same task may be associated with different sleep stages. The present study assesses trained meditating participants who are known to differ on a key factor that may be critical for procedural learning — body awareness — and compares sleeprelated changes of this group with a matched, meditation-naïve, control group.

\section{Sleep spindles}

One reliable index of memory consolidation in NREM sleep is the sleep spindle, a phasic event (.5- to 3.0 -sec duration) characterized by bursts of $11-16 \mathrm{~Hz}$ EEG activity occurring predominantly during stage 2 NREM (N2) sleep. Converging evidence points to possible different neural mechanisms implicated in fast and slow sleep spindles [68], but few studies to date have examined the combined contribution of slow and fast sleep spindles to memory consolidation. In one recent study, fast (but not slow) sleep spindles were involved in transfer or implicit knowledge to more explicit awareness [69]. In another study, consolidation of face memory, which can be considered 
as having both explicit and implicit components, was associated with both fast and slow sleep spindles [70]. In this case, fast spindles showed a positive correlation with post-sleep face recognition, and slow spindles showed a negative correlation.

The current study focuses on procedural memory, specifically on motor skill acquisition of a balance-oriented task. Procedural memory is thought to be acquired though repetition of a skilled activity that is stabilized over time [71]. However, little is known about the effect of sleep on balance. Sleep deprivation was reported to negatively affect postural stability [72-74], but no study to our knowledge has assessed relative contributions of sleep stages and sleep spindles on a postural task.

\section{Meditation and heightened body awareness}

Recent years have seen a significant increase in research on the effects of various meditation practices, often grouped under the umbrella of "mindfulness" practices, on neuroplasticity, attention, and emotion regulation processes among others. While all contemplative practices share some elements such as attention training, body awareness [7] and emphasis on insight and selfregulation [75], different meditation practices are characterized by different kinds of subjective experiences, recruit different neural networks and may have different effects on cognitive and physical functions, including well-being $[76,77]$.

Vipassana meditation is characterized by developing a sustained and systematic practice of being aware of one's bodily sensations in order to ultimately gain insight into the nature of reality [78-80]. Practitioners typically start with a focused attention practice, specifically with mindfulness of breathing or anapana. The main part of the practice consists of the body scanning technique, where practitioners are instructed to mentally examine their bodily states to become aware of subtle sensations and, ultimately, to approach them with equanimity and nonjudgementally. We hypothesize that this contemplative tradition may change the practitioner's cognitive style in a 
global way, including changing processes of attention, memory encoding, consolidation and retrieval.

Recent research showed that Vipassana practice is similar to other contemplative practices in having the beneficial effects of decreasing stress and improving subjective feelings of well-being [81], helping generate greater perceptual clarity and decrease automated reactivity to stimuli [8284], and decreasing anger, hostility and depressive symptoms [85]. Other studies report improved psychological well-being [86] and cognitive flexibility [85] and increased heart rate variability [87]. Other effects of Vipassana training include increased somatosensory awareness, e.g., increased awareness of pain, more spontaneous body movements, increased mindfulness, and development of equanimity, or the ability to adapt to extreme changes in lived experience [88].

Sleep architecture changes of Vipassana practitioners are few, but include longer REM sleep periods when compared to controls and yoga practitioners [89]. Longer-term practitioners (over 7 years of daily practice) have more N3 sleep, less N2 sleep, fewer awakenings from sleep and an altered pattern of REM sleep microarchitecture [90].

While the idea that meditation practice, by virtue of enhancing specific cognitive and attentional skills [91-93], should have an effect on learning and memory seems intuitive, very few studies to date have directly addressed the effect of meditation on memory consolidation. And existing results are mixed. One study reports very few differences between long-term meditation practitioners (including Vipassana meditators) and non-meditating controls; meditators showed better performance on short-term and free recall long-term memory [94]. Some studies report improvements in working memory in meditation practitioners [95], including a military cohort [96], and adolescents [97].

With respect to procedural motor memory, only one study to our knowledge assessed contribution of post-training meditation practice to memory consolidation [98]; experienced yoga nidra meditation practitioners showed post-training memory benefits on a sequence tapping task. 
In sum, Vipassana meditation may produce changes in body awareness, which in turn may influence not only health and well-being but the effectiveness of memory. While studies of procedural memory are lacking, the preceding review suggests that increased body awareness may help meditators excel at procedural tasks. Critically, there are as yet no studies of sleep-dependent learning in meditators.

\section{Objectives and hypotheses:}

The primary objective of the present study was to investigate the relationship between Vipassana meditation practice and sleep-dependent processes of memory consolidation. Specifically, we tested the idea that training in a meditation technique which enhances introspective accuracy and increases body awareness will influence sleep characteristics and style of sleep-dependent learning of a procedural full-body task.

1) following a daytime nap Vipassana meditators (MED group) will show more improvement on a procedural task than will meditation-naïve controls (CTL group).

2) the MED and CTL groups will express two distinct neurobiological learning patterns:

a. the MED group will show a NREM-dependent pattern, reflecting a more explicit learning process. fast sleep spindles in particular will correlate with task improvement

b. the CTL group will show a REM-dependent pattern, consistent with previous research on non-declarative learning.

\section{Methods}

\section{Participants}

Forty-two male $(n=21)$ and female $(n=21)$ participants between the ages of 18 and 35 y $(M=$ $25.4 \pm 4.4$ ) were recruited for a daytime nap study via online advertisements and through contact with Vipassana Quebec and subsequently screened via phone or online questionnaire. In the 
Vipassana meditation practitioners group (MED) there were 22 participants (M age $=25.8 \pm 4.1,11$ men, 11 women) and in the non-meditating control group (CTL) there were 20 participants (M age $=25.0 \pm 4.8,10$ men, 10 women). Inclusion criteria for both groups were: 18-35 years of age, high dream recall (3+ per week), good self-reported physical/mental health, no sleep disturbances (e.g., shift work or jet lag). To be included in the MED group, Vipassana practitioners had to have taken part in at least one 10-day retreat, which consists of approximately 100 hours of practice, and to be currently practicing Vipassana meditation on at least a weekly basis. Participants completed an informed consent form approved by the Hôpital du Sacré-Coeur de Montréal ethics board. They were financially compensated for the time spent in the laboratory, parking or public transit, and lunch expenses.

\section{Body Awareness}

Body awareness was measured using the Scale of Body Connection (SBC) questionnaire [99], which consists of 20 items each scored on 0-4 scales and which produces two independent subscales: body awareness (12 items) with items that assess awareness of, e.g., tension, bodily stress, breath, and emotional state, and bodily dissociation ( 8 items) with items that assess e.g., feeling frozen/numb, separated from the body, and difficulty in expressing emotions. Subscale scores consist of the average ratings of constituent items (0-4). The questionnaire has been validated on studies of bodily awareness in women with substance abuse disorder [100], of exercise and body awareness therapy in major depression [101], of body therapy for survivors of childhood sexual abuse [102], and of interoceptive awareness of breathing in experienced meditation practitioners [103]. This and the following questionnaires were completed at home and sent back to the laboratory by mail: short versions of the Beck Depression Inventory, Beck Anxiety Inventory, and Boundary Questionnaire; full versions of the Dream Experience Questionnaire; Mirror Behaviour Questionnaire; Dream Enactment Behaviour; Toronto Alexithymia Scale; Other Experiences Questionnaire. Results from these questionnaires are not discussed further in this paper. 


\section{Procedure}

Participants arrived in the laboratory at 9:00 am and completed a consent form and a battery of questionnaires which included the Scale of Body Connection. They then completed the procedural task and had the PSG setup installed. Immediately before lights-out, the MED group meditated in bed for 10 minutes and the CTL group simply relaxed for the same amount of time. All participants were then given a window of opportunity to sleep of approximately 90 minutes. They were awakened twice with a non-stressful, $80 \mathrm{~dB}, 500 \mathrm{~Hz}$, once at sleep onset and once at the end of the nap. At these times, spontaneous dream reports and dream questionnaires were completed. Following the second awakening, electrodes were removed and participants repeated the procedural task.

\section{Procedural memory (balance) task}

Participants performed a procedural memory task requiring whole body balance, i.e., a video game entitled "Balance bubble" for the Nintendo Wii Fit Balance Board. In this task, participants control a virtual character that moves along a river in a bubble by shifting their weight on the Balance Board. The objective is to complete the river path as quickly as possible without touching the river's edges and thus bursting the bubble. Bursting the bubble required the participant to start again from the beginning of the path. The task was performed on a 42-in. television screen. All participants were assessed for any vision correction and instructed to wear their glasses or contact lenses if needed. Participants had a 2-minute period to acquaint themselves with the task, and to ask questions of the experimenter, who ensured that participants clearly understood the requirements of the game. The total maximum game time was 90 seconds, and participants were allowed to repeat the task until a total of 5 minutes of gameplay had elapsed. Participants were instructed to attempt to complete the task as many times as possible during the 5-minute period. The task was performed once before lights-out (T1) and once after awakening (T2). 
The following variables were calculated to assess performance on the task: HiScore (highest score on all attempts averaged); \#Wins (the number of times participants completed the task by arriving at the end of the "river"); GameScore (average score in game "meters" over all attempts at the game) and Time (average time spent correctly balanced in the bubble averaged over all attempts). Two measures of improvement on the task were used: T2-T1GameScore (Score after nap minus score before nap), and T2-T1Time (Time after nap minus TimeBalance before nap).

\section{Polysomnography}

Participants slept in a quiet bedroom under continuous audio-visual monitoring with a two-way intercom. A standard polysomnographic montage was used: 6 EEG channels (F3, F4, C3, C4, O1, O2) referenced to A1 and re-referenced to A1+A2 offline; four electrooculography (EOG) channels (horizontal and vertical), and three electromyography (EMG) channels (chin, wrist, leg). Acquisition of EEG signals was done using an M15 Grass Acquisition System ( -6 dB filters with cut-offs at 0.30 and $100 \mathrm{~Hz}$ ) and Harmonie v6.2b software (Natus Medical Incorporated, Pleasanton, CA, USA). Sleep stages were scored according to standard criteria [104] by an experienced technician and standard sleep variables were calculated using in-house software.

Sleep spindles were detected on artifact-free sleep epochs recorded from F3, F4, C3, C4, O1 and $\mathrm{O} 2$ derivations by an in-house detector. The full detection algorithm is described by Nielsen et al [105] and by O'Reilly and Nielsen [106]. Spindles were separated into slow (10.00-12.99 Hz) and fast $(13.00-16.00 \mathrm{~Hz})$ types and densities calculated as the number of spindles of each type divided by time elapsed in $\mathrm{N} 2$ sleep.

\section{Results}

\section{Body awareness and dissociation}

32 participants (15 MED and $17 \mathrm{CTL} ; 80 \%$ ) returned questionnaires by mail. Independent samples t-tests on the SBC score and Body Awareness and Bodily Dissociation subscales revealed, as predicted, that the MED group scored significantly higher on Body Awareness than did the CTL 
group $(\mathrm{M}(\mathrm{MED})=.243 \pm .060 ; \mathrm{M}(\mathrm{CTL})=.194 \pm .061 ; \mathrm{t}(30)=2.289 ; \mathrm{p}=.029,2$-tailed $)$ but did not differ on Bodily Dissociation.

Body Awareness and Bodily Dissociation scores were not correlated in the MED group $(r=.182$, $\mathrm{p}=.517)$ but tended to be inversely correlated $(r=-.475, \mathrm{p}=.054)$ in the CTL group. Body Awareness and Bodily Dissociation were not correlated with years of meditation experience in the MED group (Body Awareness: $r=-.471, p=.077$; Bodily Dissociation: $r=.079, p=.779$ ).

The MED group showed positive correlations between Body Awareness and average time $(r=.580 ; \mathrm{p}<.05)$ and score $(r=.518 ; \mathrm{p}<.05)$ on the task at $\mathrm{T} 1$, and a strong negative correlation between Bodily Dissociation and task improvement $(r=-.546 ; \mathrm{p}<.05)$. In contrast, CTL participants showed a different pattern: no significant correlations were observed between Body Awareness scores and task performance measures but strong positive correlations were seen between Bodily Dissociation and several task measures: average time in balance at T2 $(r=.681 ; \mathrm{p}<.01)$; average score at $\mathrm{T} 2(r=.530 ; \mathrm{p}<.05)$; average time improvement $(r=.592 ; \mathrm{p}<.05)$; and average score improvement $(r=.619 ; \mathrm{p}<.01)$. 


\section{Task performance}

Separate paired samples t-tests were used to evaluate whether groups improved on the task following the nap. Scores on average time spent balanced on the board (T2-T1Time), average (GameScore) and highest score (HiScore) before nap (T1) and after nap (T2) served as improvement measures. Both MED and CTL groups significantly improved on all three measures (see Table 1/Figure 1).

\section{Insert Figure 1 about here \\ Insert Table 1 about here}

No group differences were observed on post-nap measures of task improvement. However, meditators finished the game more often (more \#Wins), especially after the nap: 2 (10\%) of CTL participants finished the task at least once before the nap (T1), compared with 5 (25\%) of MED participants $(\chi 2=0.693, p=.405$, 2-tailed, Yates corrected) whereas 6 (30\%) of CTL participants finished the task after the nap (T2) compared with $12(60 \%)$ of MED participants $(\chi 2=3.636$, $\mathrm{p}=.057)$ (Figure 2).

Insert Figure 2 about here

Similarly, the HiScore at T1 was significantly higher in MED than in CTL $(\mathrm{M}(\mathrm{MED})=1028.4 \pm 142.21 ; \mathrm{M}(\mathrm{CTL})=913.3 \pm 187.54 ; \mathrm{t}(38)=2.188 ; \mathrm{p}=.035)$. For a comparison of complete results on task performance between the two groups see Table 1.

Insert Table 2 about here

\section{Sleep structure}

Two MED participants were dropped from spindle analyses due to insufficient sleep duration. No statistically significant differences between MED and CTL groups were observed for 
any sleep characteristic, except for a non-significant trend $(p=.09)$ for the MED group to have lower total sleep duration (See Table 3 for complete results).

Insert Table 3 about here

\section{Relationship between sleep and meditation experience}

For the MED group, Spearman correlations revealed a strong negative correlation between lifetime meditation experience (in hours) and time in NREM2 sleep $\left(r_{\mathrm{s}}=-.546, p=.015\right)$ but no other relationships with sleep measures.

\section{Sleep spindles}

A total of $20 \mathrm{CTL}$ and $20 \mathrm{MED}$ subjects were compared (Figure 3 for group comparisons of spindle density). There were no group differences in total spindle density or fast spindle density. However, the MED group showed lower slow spindle densities in occipital derivations, specifically: a lower spindle density in $\mathrm{O} 1(\mathrm{M}(\mathrm{MED})=1.074 \pm .797 ; \mathrm{M}(\mathrm{CTL})=1.754 \pm .100 ; \mathrm{t}(38)=2.385 ; \mathrm{p}=.022)$ and a marginally lower slow spindle density in $\mathrm{O} 2(\mathrm{M}(\mathrm{MED})=1.264 \pm .838 ; \mathrm{M}(\mathrm{CTL})=1.848 \pm 1.035$; $\mathrm{t}(38)=1.963 ; \mathrm{p}=.057)$.

\section{Insert Figure 3 about here}

\section{Sleep and procedural memory consolidation}

To assess sleep relationships with improvement on the balance task, the two most sensitive task measures were used: change in average time spent in balance (T2-T1) and change in average score (T2-T1). Spearman correlations were calculated to evaluate dose-response relationships between change in sleep stages, task performance, and slow and fast spindle density.

\section{Sleep stages}

For the MED group there were no statistically significant relationships between task improvement scores (average time and average score) and sleep stages. For CTL group, 
improvement on average time spent balanced correlated negatively with Stage 1 NREM duration $(r=.-465, \mathrm{p}=.007)$ and positively with REM sleep duration $(r=.592, \mathrm{p}=.006)$. Further, improvement on average score correlated negatively with Stage1 NREM duration $(r=-.470, \mathrm{p}=.037)$; and positively with REM sleep duration $(r=.536, \mathrm{p}=.015)$. Improvement on average score also correlated with several other sleep measures which were not predicted, i.e., negatively with sleep latency $(r=-.475, \mathrm{p}=.034)$; positively with sleep efficacy $(r=.451, \mathrm{p}=.046)$; and negatively with wake duration $(r=-.498, \mathrm{p}=.025)$. See Table 4 for complete results.

Insert Table 4 about here

\section{Sleep spindles}

Pearson correlations between measures of task improvement (T2-T1Time and T2T1GameScore) and sleep spindle densities revealed significant relationships for the MED group, but not the CTL group (Figure 4). T2-T1Time correlated positively with density of slow sleep spindles in $\mathrm{O} 1(r=.515, \mathrm{p}=.024)$ and marginally in $\mathrm{O} 2(r=.421 ; \mathrm{p}=.072)$. T2-T1GameScore correlated positively with density of slow sleep spindles in O1 ( $r=.539$, p-.017); negatively with density of fast sleep spindles in $\mathrm{O} 1(r=-.551, \mathrm{p}=.014)$; and negatively with density of fast sleep spindles in $\mathrm{O} 2(r=-.520, \mathrm{p}=.022)$.

\section{Insert Figure 4 about here}

\section{Discussion}

This study is the first to examine sleep-dependent memory consolidation in meditation practitioners. Although meditators were found to perform slightly better on the balance task at first testing than were controls, both groups improved to a similar extent after a morning nap. However, distinct patterns of relationships between task improvement and sleep structure were observed for the two groups: meditators showed a relationship between task improvement and occipital NREM sleep spindles while controls showed a relationship between task improvement and REM sleep time. Additionally, there were marked differences in spindle density between meditators and controls, with meditators having significantly lower spindle density, especially in occipital regions. 
These results together suggest that meditation experience may contribute to large-scale changes in the electrophysiological indicators of neuroplasticity during sleep.

\section{Different sleep-dependent memory consolidation styles in meditators and controls}

Results support the study's main hypothesis that Vipassana meditation practitioners, by virtue of training in attending to bodily states and stimuli, may rely upon a different neurobiological learning style, one that manifests in distinct changes in sleep microarchitecture.

Specifically, meditation practitioners showed relationships between the density of slow and fast occipital spindles in N2 sleep and task improvement. While slow occipital spindle density correlated positively with task improvement, fast occipital spindle density showed the opposite. Meditators did not, however, show any links between duration of REM or NREM sleep stages and task improvement. On the other hand, non-meditating controls did show a strong positive correlation between time in REM sleep and task improvement. This is consistent with previous research where in a series of studies a novel motor skill, in this case jumping on a trampoline, was associated with increased REM sleep $[39,40]$. Our results partially replicate these findings to the extent that improvement on our procedural balance task was associated with length of REM sleep during the daytime nap for control participants only. They did not, however, show any links between sleep spindles, especially fast sleep spindles, and task improvement. Both of these findings have been reported in previous studies. To illustrate, for the N2 spindle finding among meditators, we previously reported similarly perpendicular relationships between fast and slow spindles in relation to learning: fast N2 spindles correlated positively and slow spindles correlated negatively with face recognition [70]. For the REM sleep finding in controls, earlier research similarly linked duration of REM sleep with implicit memory consolidation [60].

In sum, post-task sleep architecture of meditators was distinctly different from that of nonmeditating controls; it was correlated with occipital spindle density in N2 sleep but not with the duration of any sleep stages whereas in controls task improvement was associated with increased time in REM sleep, but not with N2 sleep spindles. Both patterns of sleep changes have been 
observed in prior research although never linked differentially to meditation experience as in the present study.

\section{Different styles of post-task sleep-dependent processing?}

One interpretation of these findings is that meditation practitioners approach a procedural fullbody balance task in a more "explicit" manner, in the sense that they are able to bring to awareness elements of their somatic and bodily experience that usually remain unconscious among untrained individuals. Their greater familiarity with bodily sensations may thus have allowed them to perform somewhat better on some, but not all, aspects of the task. They performed slightly better than the control group on the task overall, achieving higher maximum scores both before and after the nap. This advantage may reflect a shift in the neuroplasticity mechanisms that were brought to bear on the task during sleep. This idea is consistent with the suggestion (Smith, Aubrey and Peters [107] that REM sleep is associated with processing of complex tasks while Stage 2 sleep benefits the consolidation of simpler tasks. Smith et al (2004) propose that the involvement of REM or Stage 2 sleep in consolidation of a motor task depends on perceived complexity of the task; participants who are less skillful at the outset will show a dependence on REM sleep and those who find the task easier will show a N2 dependence. Because our meditators scored higher in body awareness and possibly found the task slightly easier, they may have needed only N2 spindle mechanisms to refine their learning. In contrast, if control participants found the task more complex or novel they may have had a greater need for REM sleep control mechanisms.

Despite the feasibility of this explanation, the significance of several additional findings from the current study remain unclear. One is the question of why meditators had a lower overall rate of spindles when sleep spindle density was associated with task improvement.

A second outstanding question is why slow rather than fast sleep spindle densities were associated with task improvement. Many previous studies [63, 108, 109] report that fast spindle density is preferentially correlated with improvement on procedural tasks. However, a growing body of evidence supports the notion that slow spindles are also a reliable correlate of procedural 
learning in some contexts. Nishida and colleagues [110] showed a negative association between slow spindle power and a finger-tapping motor tracing task in depressed but not in healthy participants. A second group [30] showed a positive correlation between slow spindle activity (sigma: $12-14 \mathrm{~Hz}$ ) in NREM sleep and improvement on a mirror tracing task. Third, some studies have shown that slow spindles are associated with explicit memory consolidation on tasks such as word-lists [30] and auditory verbal learning and declarative learning in epileptic patients [111]. Fourth, in children slow sleep spindle activity was found to be associated with declarative learning efficiency [112]. Finally, in a study using daytime naps [113], participants learning difficult and easy word-pairs showed evidence of slow spindle associations with improvement only on the difficult pairs. Such converging evidence links slow spindles to procedural learning and is consistent with our finding that meditators reacted to the balance task as a procedural task requiring N2 sleep mediation.

\section{Possible napping profile}

A second possible explanation of our group differences in sleep-dependent effects is that meditators are conforming to a profile known to characterize habitual nappers, either because they are used to taking frequent naps or because periods of daytime meditation may confer advantages similar to those provided by sleep, especially theta-rich Stage 1 NREM sleep [114]. Unfortunately, our findings do not further clarify the first point because we did not collect information about habitual napping practices. Yet, there is evidence [115] that individuals habitually taking daytime naps show a relationship between motor task improvement and sleep spindles whereas non-habitual nappers do not. Such findings, together with evidence that meditation practitioners commonly fall asleep during meditation sessions [116], provides some support for the notion that meditation confers either sleep-dependent or sleep-like advantages in memory consolidation. However, more research taking into account the habitual napping practices of participants is clearly needed for this explanation to be considered probable. 
To summarize, our finding of different patterns of sleep-dependent memory consolidation for meditating and non-meditating controls can be accounted for by at least two explanations. On the one hand, the body-focused practices of meditation may contribute to large-scale changes in learning, including changes in sleep microarchitecture and sleep-dependent memory consolidation. On the other hand, similarities between meditation practice and habitual napping may confer additional sleep-related or sleep-like advantages to meditators. At present, more research supports the former possibility as an explanation for the present findings.

The notion that meditation practice leads to a different style of learning is consistent with both the philosophy of meditation approaches and a growing body of research. Indeed, the underlying goal of most traditional meditation practices, including Vipassana meditation, is not simple training of attentional acuity, but rather development of insight into one's own patterns of reactivity in order to decrease unwholesome traits and increase wholesome traits and behaviours $[79,80]$. Ultimately, the goal is not to improve at meditation, but to improve at life skills more generally. Thus, neuroplastic changes and acquired mental skills associated with meditation practices may have an important effect on changing one's cognitive, emotional and memory patterns more broadly [117]. This idea is consistent with previous findings, including those from a study wherein mindfulness training led to increased somatosensory awareness of the experience of sadness, which in turn was related to decreased depression [118]. Neurophysiologically, meditation training may change the functional connectivity associated with bodily representation and thereby recruit the interoceptive skills that underlie several perceptual and cognitive tasks [119]. Our findings are thus consistent with the notion that Vipassana practice, through increased interoception and body awareness, facilitates the development of cognitive skills and thereby differentially influences sleep's reactions to new procedural learning tasks.

\section{Task performance, body awareness and bodily dissociation}

Both meditators and non-meditating controls improved their performance on the balance task following a daytime nap. Contrary to our predictions, meditators performed only marginally better 
on the task than did controls: they did not improve more than controls overall, but they had higher scores before the nap and they completed the task more often after the nap.

As predicted, however, meditators scored higher than controls on the Body Awareness subscale of the Scale of Body Connection and that these scores were related in a graded fashion to task performance (time and score) before the nap. These finding are consistent with studies suggesting that sustained meditation practice contributes to higher levels of somatic awareness [8] and confirms our rationale for choosing a full-body balance task to examine patterns of sleep-dependent memory consolidation. In this case, one required skill for learning the task quickly is a heightened level of attunement to one's own bodily/somatic states.

\section{Sleep and spindle differences between meditators and controls}

\section{Sleep architecture}

In contrast to existing literature, we did not find longer REM sleep or SWS periods in meditation practitioners or differences in sleep efficiency or \#awakenings, but meditators did show a tendency for a lower overall sleep time. For instance, in one study on Mindfulness-Based Cognitive Therapy, meditation practitioners showed more cortical arousal and more awakenings during nighttime sleep than did controls [120]. On the other hand, experienced long-term Buddhist practitioners [121] had a lower total sleep time like the trend in our study but also more awakenings — unlike our sample.

The fact that we found no associations between meditation experience and sleep latencies, sleep efficiency or duration/proportion of individual sleep stages may be due to the fact that our sample contained no expert meditation practitioners, typically quantified as having more than 10000 hours of meditation practice [122]. Because all our participants can be considered meditation novices or at best intermediate practitioners, it is possible that the short daytime naps taken were insufficiently sensitive to reveal subtle group differences. Future studies might require all-night recordings or protocols that unmask subtle group differences by augmenting homeostatic pressure or REM sleep propensity. 


\section{Sleep spindles}

We found sleep spindle density differences between meditators and controls, with meditators unexpectedly showing reduced sleep spindle density, especially in occipital derivations. Reduced spindle density has been suggested as a marker of neurodegeneration $[123,124]$ and psychopathological conditions such as schizophrenia $[18,125]$. Our finding is unexpected because practice of meditation has been associated with increased neuroplasticity [126-128], and is thought to have neuroprotective effects against cognitive decline [129] and psychopathology [130]. For instance, in one study [131] Vipassana practitioners showed no age-related decline in slow-wave sleep and REM sleep compared to controls, suggesting that Vipassana practice may protect against age-related neurodegeneration.

Sleep spindles have also been suggested to play a role in maintaining sleep in face of environmental stressors, especially noise [132-135]. Reduced sleep spindles, in addition to other markers of physiological arousal during sleep in meditation practitioners may represent a general developed trait of increased alertness/awareness of the environment during sleep. While in normal populations sleep fragmentation may represent a pathological hyper-vigilance that may lead to insomnia and increased stress levels $[136,137]$ in meditation practitioners, it may not have the same negative effect due to practices that emphasize non-reactive awareness, an active monitoring of the contents of awareness, and a general increase in alertness [138]. These effects, however, tend to depend on proficiency in meditation practice: meditators in early stages of practice report more fatigue and sleepiness, while more experienced practitioners report greater alertness [139].

Brain synchronization mechanisms have been proposed to be a marker of neuroplasticity, reflecting processes of strengthening of neuronal connections and creation of new, experiencedependent, networks [140]. These processes were shown to have a strong top-down attentional component [141]. Long-term meditation practitioners generate long-distance phase-synchrony during a compassion meditation practice, and express EEG signatures different from those of controls during both active practice and the resting state [142]. This long-distance synchrony has been proposed to be a marker of cognitive integration [143, 144], and alterations in neural 
synchrony patterns can be seen as markers of psychopathology [145]. Sleep spindles involve synchronization on the level of thalamo-cortical communication in NREM sleep [146]. Recently, slow spindles were reported to be associated with brain synchrony over longer circuits, and faster spindles with more local connectivity [147]. Thus, involvement of slow sleep spindles in procedural memory consolidation in meditation practitioners may reflect large-scale reorganization of brain connectivity—perhaps an increased efficiency of these networks — which may positively affect many cognitive skills, including memory.

Meditation practitioners, therefore, may develop a trait-like neurophysiological profile that involves both daytime and sleep changes. This trait may be similar to that of individuals with high dream recall: in a recent study we showed that increased fast but decreased slow sleep spindles correlate with dream recall frequency [105]. The latter is a measure previously linked with higher reactivity to auditory stimuli both in wake and during sleep [148], and with increased activity in the temporoparietal junction and medial prefrontal cortex - areas typically associated with attention and memory [149]. This finding is consistent with observations in our current study: all our participants had high dream recall, and meditators had fewer slow sleep spindles than did non-meditating controls, possibly reflecting their increased alertness in sleep.

Another possible explanation of our group difference in slow spindles is that some effects of meditation practice overlap with the function of sleep spindles, thus reducing the processing load on sleep-dependent processes. For instance, sleep spindles are associated with intelligence [150-153], and pharmacologically induced sleep spindles correlate with memory for emotionally negative memory, suggesting an emotion regulation function [154]. Analagously, meditation practice may contribute to the slowing of age-related decline in fluid intelligence [155], to improvement in cognitive function [93] and to enhanced emotion regulation [156].

Finally, it is possible that meditation subsumes some of the beneficial functions of sleep. While meditation practice is generally associated with increased self-reported well-being, health and life quality, during intensive meditation practice e.g., a meditation retreat, meditators require less sleep 
[88], and exhibit physiological arousal signs, such as increased gamma coherence during slow wave sleep [121]. And while sleep plays an important role in memory consolidation, restful wakefulness (as opposed to active wakefulness) also promotes learning [157]. It is possible, therefore, that daytime meditation practice is sleep-like in nature and thus reduces sleep pressure and alter subsequent sleep architecture.

\section{Acknowledgements}

This research and ES were funded by the Francisco J. Varela Research Award from the Mind and Life Institute, by the Joseph-Armand Bombardier doctoral scholarship from the Social Science and Humanities Research Council of Canada, by the J.-A. De Sève doctoral scholarship from the Fondation de l'Hôpital du Sacré-Coeur de Montréal and by an Operating Grant from the Natural Sciences and Engineering Research Council of Canada to TN. CB-C was funded by a master's scholarship from the Canadian Institutes for Health Research. The authors are grateful to Alexandra Duquette, Olivier Dussault, Sarah Hertz, Cecile Renucci, and Jonathan Faucher for help in development of the experimental protocol, pilot work, and analyses of dream reports.

\section{Author contributions}

ES and TN designed the study, interpreted the results and wrote the manuscript and ES, TN, SD, AS-R, CB-C, MC, TP collected data and analyzed results.

\section{References}

1. Varela, F.J., E. Thompson, and E. Rosch, The Embodied Mind: Cognitive Science and Human Experience. 1992, Cambridge: MIT Press.

2. Thompson, E., Sensorimotor subjectivity and the enactive approach to experience. Phenomenology and the Cognitive Sciences, 2005. 4(4): p. 407-427.

3. Thompson, E. and F.J. Varela, Radical embodiment: neural dynamics and consciousness. Trends in cognitive sciences, 2001. 5(10): p. 418-425.

4. Gallagher, S., How the body shapes the mind. 2005: Cambridge Univ Press. 
5. Schmalzl, L., M.A. Crane-Godreau, and P. Payne, Movement-based embodied contemplative practices: definitions and paradigms. Front Hum Neurosci, 2014. 8: p. 205.

6. Zeng, X., T.P. Oei, and X. Liu, Monitoring emotion through body sensation: a review of awareness in Goenka's Vipassana. J Relig Health, 2014. 53(6): p. 1693-705.

7. Kerr, C.E., et al., Mindfulness starts with the body: somatosensory attention and top-down modulation of cortical alpha rhythms in mindfulness meditation. Front Hum Neurosci, 2013. 7: p. 12.

8. Fox, K.C., et al., Meditation experience predicts introspective accuracy. PLoS One, 2012. 7(9): p. e45370.

9. Mehling, W.E., et al., Body awareness: a phenomenological inquiry into the common ground of body-mind therapies. Philosophy, Ethics, and Humanities in Medicine, 2011. 6(6): p. 1-12.

10. Silverstein, R.G., et al., Effects of mindfulness training on body awareness to sexual stimuli: implications for female sexual dysfunction. Psychosom Med, 2011. 73(9): p. 817-25.

11. Bornemann, B., et al., Differential changes in self-reported aspects of interoceptive awareness through 3 months of contemplative training. Front Psychol, 2014. 5: p. 1504.

12. Graf, P. and D.L. Schacter, Implicit and explicit memory for new associations in normal and amnesic subjects. J Exp Psychol Learn Mem Cogn, 1985. 11(3): p. 501-18.

13. Squire, L.R., Memory systems of the brain: a brief history and current perspective. Neurobiol Learn Mem, 2004. 82(3): p. 171-7.

14. Tulving, E., Episodic memory: from mind to brain. Annu Rev Psychol, 2002. 53: p. 1-25.

15. Baars, B.J., The conscious access hypothesis: origins and recent evidence. Trends Cogn Sci, 2002. 6(1): p. 47-52.

16. Schacter, D.L., C.-Y.P. Chiu, and K.N. Ochsner, Implicit memory: a selective review. Annual Review of Neuroscience, 1993. 16(1): p. 159-182.

17. Antonenko, D., et al., Napping to renew learning capacity: enhanced encoding after stimulation of sleep slow oscillations. Eur J Neurosci, 2013. 37(7): p. 1142-51.

18. Wamsley, E.J., et al., Reduced sleep spindles and spindle coherence in schizophrenia: mechanisms of impaired memory consolidation? Biol Psychiatry, 2012. 71(2): p. 154-61.

19. Holz, J., et al., The timing of learning before night-time sleep differentially affects declarative and procedural long-term memory consolidation in adolescents. PLoS One, 2012. 7(7): p. e40963.

20. Genzel, L., et al., Sleep-dependent memory consolidation of a new task is inhibited in psychiatric patients. J Psychiatr Res, 2011. 45(4): p. 555-60.

21. Dresler, M., et al., Impaired off-line memory consolidation in depression. Eur Neuropsychopharmacol, 2010. 20(8): p. 553-61.

22. Van Der Werf, Y.D., et al., Learning by observation requires an early sleep window. Proc Natl Acad Sci U S A, 2009. 106(45): p. 18926-30.

23. Doyon, J., et al., Contribution of night and day sleep vs. simple passage of time to the consolidation of motor sequence and visuomotor adaptation learning. Exp Brain Res, 2009. 195(1): p. 15-26. 
24. Benedict, C., et al., Enhancing influence of intranasal interleukin-6 on slow-wave activity and memory consolidation during sleep. FASEB J, 2009. 23(10): p. 3629-36.

25. Ertelt, D., et al., Skill memory escaping from distraction by sleep--evidence from dual-task performance. PLoS One, 2012. 7(12): p. e50983.

26. Prehn-Kristensen, A., et al., Sleep restores daytime deficits in procedural memory in children with attention-deficit/hyperactivity disorder. Res Dev Disabil, 2011. 32(6): p. 2480-8.

27. Galea, J.M., et al., Disruption of the dorsolateral prefrontal cortex facilitates the consolidation of procedural skills. J Cogn Neurosci, 2010. 22(6): p. 1158-64.

28. Manoach, D.S., et al., Reduced overnight consolidation of procedural learning in chronic medicated schizophrenia is related to specific sleep stages. J Psychiatr Res, 2010. 44(2): p. 112-20.

29. Tucker, M.A. and W. Fishbein, The impact of sleep duration and subject intelligence on declarative and motor memory performance: how much is enough? J Sleep Res, 2009. 18(3): p. 304-12.

30. Holz, J., et al., EEG Sigma and slow-wave activity during NREM sleep correlate with overnight declarative and procedural memory consolidation. J Sleep Res, 2012. 21(6): p. 612-9.

31. Puetz, J., et al., Impaired memory consolidation during sleep in patients with functional memory disorder. Biol Psychol, 2011. 86(1): p. 31-8.

32. Javadi, A.H., V. Walsh, and P.A. Lewis, Offline consolidation of procedural skill learning is enhanced by negative emotional content. Exp Brain Res, 2011. 208(4): p. 507-17.

33. Smith, C.T., M.R. Nixon, and R.S. Nader, Posttraining increases in REM sleep intensity implicate REM sleep in memory processing and provide a biological marker of learning potential. Learn Mem, 2004. 11(6): p. 714-9.

34. Wilhelm, I., et al., Sleep-dependent consolidation of procedural motor memories in children and adults: the pre-sleep level of performance matters. Dev Sci, 2012. 15(4): p. 506-15.

35. Cipolli, C., et al., Sleep and time course of consolidation of visual discrimination skills in patients with narcolepsy-cataplexy. J Sleep Res, 2009. 18(2): p. 209-20.

36. Gais, S., et al., Visual-procedural memory consolidation during sleep blocked by glutamatergic receptor antagonists. J Neurosci, 2008. 28(21): p. 5513-8.

37. Suzuki, H., et al., Alpha activity during rem sleep contributes to overnight improvement in performance on a visual discrimination task. Percept Mot Skills, 2012. 115(2): p. 337-48.

38. Tjernstrom, F., P.A. Fransson, and M. Magnusson, Improved postural control through repetition and consolidation. J Vestib Res, 2005. 15(1): p. 31-9.

39. Buchegger, J., et al., Does Trampolining and Anaerobic Physical Fitness Affect Sleep? Percept Mot Skills, 1991. 73(1): p. 243-252.

40. Buchegger, J. and A. Meier-Koll, Motor learning and ultradian sleep cycle: an electroencephalographic study of trampoliners. Percept Mot Skills, 1988. 67(2): p. 635-45.

41. Wamsley, E.J., et al., Cognitive replay of visuomotor learning at sleep onset: temporal dynamics and relationship to task performance. Sleep, 2010. 33(1): p. 59-68.

42. Shönauer, M. and S. Gais, The Effect of Sleep on Multiple Systems, in Cognitive Neuroscience of Memory Consolidation. 2017, Springer International Publishing. p. 105-115. 
43. Stickgold, R., Parsing the role of sleep in memory processing. Curr Opin Neurobiol, 2013.

44. Diekelmann, S. and J. Born, The memory function of sleep. Nat Rev Neurosci, 2010. 11(2): p. 11426.

45. Walker, M.P. and R. Stickgold, Overnight alchemy: sleep-dependent memory evolution. Nat Rev Neurosci, 2010. 11(3): p. 218; author reply 218.

46. Deliens, G. and P. Peigneux, One night of sleep is insufficient to achieve sleep-to-forget emotional decontextualisation processes. Cogn Emot, 2014. 28(4): p. 698-706.

47. Rolls, A., et al., Sleep to forget: interference of fear memories during sleep. Mol Psychiatry, 2013. 18(11): p. 1166-70.

48. Stickgold, R. and M.P. Walker, Sleep-dependent memory triage: evolving generalization through selective processing. Nature Neuroscience, 2013. 16(2): p. 139-45.

49. Fogel, S.M., et al., fMRI and sleep correlates of the age-related impairment in motor memory consolidation. Hum Brain Mapp, 2014. 35(8): p. 3625-45.

50. Korman, M., Y. Dagan, and A. Karni, Nap it or leave it in the elderly: A nap after practice relaxes age-related limitations in procedural memory consolidation. Neurosci Lett, 2015. 606: p. 173-6.

51. Lahl, O., et al., An ultra short episode of sleep is sufficient to promote declarative memory performance. J Sleep Res, 2008. 17(1): p. 3-10.

52. Mednick, S., K. Nakayama, and R. Stickgold, Sleep-dependent learning: a nap is as good as a night. Nat Neurosci, 2003. 6(7): p. 697-8.

53. Seeck-Hirschner, M., et al., Effects of daytime naps on procedural and declarative memory in patients with schizophrenia. J Psychiatr Res, 2010. 44(1): p. 42-7.

54. Nishida, M. and M.P. Walker, Daytime naps, motor memory consolidation and regionally specific sleep spindles. PLoS One, 2007. 2(4): p. e341.

55. Backhaus, J. and K. Junghanns, Daytime naps improve procedural motor memory. Sleep Med, 2006. 7(6): p. 508-12.

56. Lo, J.C., D.J. Dijk, and J.A. Groeger, Comparing the effects of nocturnal sleep and daytime napping on declarative memory consolidation. PLoS One, 2014. 9(9): p. e108100.

57. Plihal, W. and J. Born, Effects of early and late nocturnal sleep on declarative and procedural memory. Journal of Cognitive Neuroscience, 1997. 9(4): p. 534-547.

58. Nielsen, T., et al., Overnight improvements in two REM sleep-sensitive tasks are associated with both REM and NREM sleep changes, sleep spindle features, and awakenings for dream recall. Neurobiol Learn Mem, 2015. 122: p. 88-97.

59. Kloepfer, C., et al., Memory before and after sleep in patients with moderate obstructive sleep apnea. J Clin Sleep Med, 2009. 5(6): p. 540-8.

60. Karni, A., et al., Dependence on REM sleep of overnight improvement of a perceptual skill. Science, 1994. 265(5172): p. 679-82.

61. Walker, M.P., et al., Practice with sleep makes perfect: sleep-dependent motor skill learning. Neuron, 2002. 35(1): p. 205-11. 
62. Fogel, S., et al., Sleep spindles: a physiological marker of age-related changes in gray matter in brain regions supporting motor skill memory consolidation. Neurobiol Aging, 2017. 49: p. 154-164.

63. Barakat, M., et al., Fast and slow spindle involvement in the consolidation of a new motor sequence. Behav Brain Res, 2011. 217(1): p. 117-21.

64. Robertson, E.M., A. Pascual-Leone, and D.Z. Press, Awareness modifies the skill-learning benefits of sleep. Curr Biol, 2004. 14(3): p. 208-12.

65. Fogel, S.M., C.T. Smith, and R.J. Beninger, Evidence for 2-stage models of sleep and memory: learning-dependent changes in spindles and theta in rats. Brain Res Bull, 2009. 79(6): p. 445-51.

66. Ficca, G. and P. Salzarulo, What in sleep is for memory. Sleep Med, 2004. 5(3): p. 225-30.

67. Fogel, S.M., et al., How to become an expert: A new perspective on the role of sleep in the mastery of procedural skills. Neurobiol Learn Mem, 2015. 125: p. 236-48.

68. Molle, M., et al., Fast and slow spindles during the sleep slow oscillation: disparate coalescence and engagement in memory processing. Sleep, 2011. 34(10): p. 1411-21.

69. Yordanova, J., et al., Sleep Spindles in the Right Hemisphere Support Awareness of Regularities and Reflect Pre-Sleep Activations. Sleep, 2017.

70. Solomonova, E., et al., Sleep-dependent consolidation of face recognition and its relationship to REM sleep duration, REM density and Stage 2 sleep spindles. Journal of Sleep Research, 2017.

71. Gupta, P. and N.J. Cohen, Theoretical and computational analysis of skill learning, repetition priming, and procedural memory. Psychol Rev, 2002. 109(2): p. 401-48.

72. Robillard, R., et al., Aging worsens the effects of sleep deprivation on postural control. PLoS One, 2011. 6(12): p. e28731.

73. Robillard, R., et al., Effects of increased homeostatic sleep pressure on postural control and their modulation by attentional resources. Clin Neurophysiol, 2011. 122(9): p. 1771-8.

74. Schlesinger, A., et al., Postural control, attention and sleep deprivation. Neuroreport, 1998. 9(1): p. 49-52.

75. Lutz, A., et al., Investigating the phenomenological matrix of mindfulness-related practices from a neurocognitive perspective. Am Psychol, 2015. 70(7): p. 632-58.

76. Fox, K.C., et al., Functional neuroanatomy of meditation: A review and meta-analysis of 78 functional neuroimaging investigations. Neurosci Biobehav Rev, 2016. 65: p. 208-28.

77. Fox, K.C., et al., Is meditation associated with altered brain structure? A systematic review and meta-analysis of morphometric neuroimaging in meditation practitioners. Neurosci Biobehav Rev, 2014. 43: p. 48-73.

78. Chavan, D.V., Vipassana: the Buddha's tool to probe mind and body. Prog Brain Res, 2008. 168: p. 247-53.

79. Goenka, S.N., The art of living: Vipassana Meditation. 1997: Vipassana Research Institute.

80. Hart, W., The art of living: Vipassana meditation as taught by S.N. Goenka. 1987: Pariyatti.

81. Szekeres, R.A. and E.H. Wertheim, Evaluation of Vipassana Meditation Course Effects on Subjective Stress, Well-being, Self-kindness and Mindfulness in a Community Sample: Post-course and 6-month Outcomes. Stress Health, 2015. 31(5): p. 373-81. 
82. Cahn, B.R. and J. Polich, Meditation (Vipassana) and the P3a event-related brain potential. Int J Psychophysiol, 2009. 72(1): p. 51-60.

83. Cahn, B.R., A. Delorme, and J. Polich, Event-related delta, theta, alpha and gamma correlates to auditory oddball processing during Vipassana meditation. Soc Cogn Affect Neurosci, 2013. 8(1): p. 100-11.

84. Delgado-Pastor, L.C., et al., Mindfulness (Vipassana) meditation: Effects on P3b event-related potential and heart rate variability. Int J Psychophysiol, 2013.

85. Kasai, Y., et al., Psychological effects of meditation at a Buddhist monastery in Myanmar. J Ment Health, 2015: p. 1-4.

86. Montero-Marin, J., et al., Psychological Effects of a 1-Month Meditation Retreat on Experienced Meditators: The Role of Non-attachment. Front Psychol, 2016. 7: p. 1935.

87. Krygier, J.R., et al., Mindfulness meditation, well-being, and heart rate variability: a preliminary investigation into the impact of intensive Vipassana meditation. Int J Psychophysiol, 2013. 89(3): p. $305-13$.

88. Kornfield, J., Intensive insight meditation: A phenomenological study. The Journal of Transpersonal Psychology, 1979. 11(1): p. 41-58.

89. Sulekha, S., et al., Evaluation of sleep architecture in practitioners of Sudarshan Kriya yoga and Vipassana meditation. Sleep and Biological Rhythms, 2006.

90. Maruthai, N., et al., Senior Vipassana Meditation practitioners exhibit distinct REM sleep organization from that of novice meditators and healthy controls. Int Rev Psychiatry, 2016. 28(3): p. $279-87$.

91. Jha, A.P., J. Krompinger, and M.J. Baime, Mindfulness training modifies subsystems of attention. Cogn Affect Behav Neurosci, 2007. 7(2): p. 109-19.

92. Valentine, E.R. and P.L.G. Sweet, Meditation and attention: A comparison of the effects of concentrative and mindfulness meditation on sustained attention. Mental Health, Religion and Culture, 1999. 2(1): p. 59-70.

93. Zeidan, F., et al., Mindfulness meditation improves cognition: evidence of brief mental training. Conscious Cogn, 2010. 19(2): p. 597-605.

94. Lykins, E.L.B., R.A. Baer, and L.R. Gottlob, Performance-Based Tests of Attention and Memory in Long-Term Mindfulness Meditators and Demographically Matched Nonmeditators. Cognitive Therapy and Research, 2012. 36(1): p. 103-144.

95. Mrazek, M.D., et al., Mindfulness training improves working memory capacity and GRE performance while reducing mind wandering. Psychol Sci, 2013. 24(5): p. 776-81.

96. Jha, A.P., et al., Examining the protective effects of mindfulness training on working memory capacity and affective experience. Emotion, 2010. 10(1): p. 54-64.

97. Quach, D., K.E. Jastrowski Mano, and K. Alexander, A Randomized Controlled Trial Examining the Effect of Mindfulness Meditation on Working Memory Capacity in Adolescents. J Adolesc Health, 2016. 58(5): p. 489-96.

98. Immink, M.A., Post-training Meditation Promotes Motor Memory Consolidation. Front Psychol, 2016. 7: p. 1698. 
99. Price, C.J. and E.A. Thompson, Measuring dimensions of body connection: body awareness and bodily dissociation. J Altern Complement Med, 2007. 13(9): p. 945-53.

100. Price, C.J., et al., Mindful awareness in body-oriented therapy as an adjunct to women's substance use disorder treatment: a pilot feasibility study. J Subst Abuse Treat, 2012. 43(1): p. 94-107.

101. Danielsson, L., et al., Exercise or basic body awareness therapy as add-on treatment for major depression: a controlled study. J Affect Disord, 2014. 168: p. 98-106.

102. Price, C.J., Dissociation reduction in body therapy during sexual abuse recovery. Complement Ther Clin Pract, 2007. 13(2): p. 116-28.

103. Daubenmier, J., et al., Follow your breath: respiratory interoceptive accuracy in experienced meditators. Psychophysiology, 2013. 50(8): p. 777-89.

104. Iber, C., The AASM manual for the scoring of sleep and associated events: rules, terminology and technical specifications. 2007: American Academy of Sleep Medicine.

105. Nielsen, T., et al., NREM sleep spindles are associated with dream recall. Sleep Spindles and Cortical Up States, 2016.

106. O'Reilly, C. and T. Nielsen, Sleep spindle detection: Automatic detection and evaluation of performance with a fine temporal resolution. Front Hum Neurosci, 2014. submitted.

107. Smith, C.T., J.B. Aubrey, and K.R. Peters, Different roles for REM and stage 2 sleep in motor learning: A proposed model. Psychologica Belgica, 2004. 44(1-2): p. 81-104.

108. Tamaki, M., et al., Activation of fast sleep spindles at the premotor cortex and parietal areas contributes to motor learning: a study using sLORETA. Clin Neurophysiol, 2009. 120(5): p. 878-86.

109. Lustenberger, C., et al., Feedback-Controlled Transcranial Alternating Current Stimulation Reveals a Functional Role of Sleep Spindles in Motor Memory Consolidation. Curr Biol, 2016. 26(16): p. 2127-36.

110. Nishida, M., Y. Nakashima, and T. Nishikawa, Slow sleep spindle and procedural memory consolidation in patients with major depressive disorder. Nat Sci Sleep, 2016. 8: p. 63-72.

111. Del Felice, A., A. Magalini, and S. Masiero, Slow-oscillatory Transcranial Direct Current Stimulation Modulates Memory in Temporal Lobe Epilepsy by Altering Sleep Spindle Generators: A Possible Rehabilitation Tool. Brain Stimul, 2015. 8(3): p. 567-73.

112. Hoedlmoser, K., et al., Slow sleep spindle activity, declarative memory, and general cognitive abilities in children. Sleep, 2014. 37(9): p. 1501-12.

113. Schmidt, C., et al., Encoding difficulty promotes postlearning changes in sleep spindle activity during napping. J Neurosci, 2006. 26(35): p. 8976-82.

114. Aftanas, L. and S. Golosheykin, Impact of regular meditation practice on EEG activity at rest and during evoked negative emotions. Int J Neurosci, 2005. 115(6): p. 893-909.

115. Milner, C.E., S.M. Fogel, and K.A. Cote, Habitual napping moderates motor performance improvements following a short daytime nap. Biol Psychol, 2006. 73(2): p. 141-56.

116. Pagano, R.R., et al., Sleep during transcendental meditation. Science, 1976. 191(4224): p. 308-10.

117. Hasenkamp, W. and L.W. Barsalou, Effects of meditation experience on functional connectivity of distributed brain networks. Front Hum Neurosci, 2012. 6: p. 38. 
118. Farb, N.A., et al., Minding one's emotions: mindfulness training alters the neural expression of sadness. Emotion, 2010. 10(1): p. 25-33.

119. Farb, N.A., Z.V. Segal, and A.K. Anderson, Mindfulness meditation training alters cortical representations of interoceptive attention. Soc Cogn Affect Neurosci, 2013. 8(1): p. 15-26.

120. Britton, W.B., et al., Polysomnographic and subjective profiles of sleep continuity before and after mindfulness-based cognitive therapy in partially remitted depression. Psychosom Med, 2010. 72(6): p. 539-48.

121. Ferrarelli, F., et al., Experienced mindfulness meditators exhibit higher parietal-occipital EEG gamma activity during NREM sleep. PLoS One, 2013. 8(8): p. e73417.

122. Brefczynski-Lewis, J.A., et al., Neural correlates of attentional expertise in long-term meditation practitioners. Proc Natl Acad Sci U S A, 2007. 104(27): p. 11483-8.

123. Ktonas, P.Y., et al., Potential dementia biomarkers based on the time-varying microstructure of sleep EEG spindles. Conf Proc IEEE Eng Med Biol Soc, 2007. 2007: p. 2464-7.

124. Latreille, V., et al., Sleep spindles in Parkinson's disease may predict the development of dementia. Neurobiol Aging, 2015. 36(2): p. 1083-90.

125. Schilling, C., et al., Fast sleep spindle reduction in schizophrenia and healthy first-degree relatives: association with impaired cognitive function and potential intermediate phenotype. Eur Arch Psychiatry Clin Neurosci, 2016.

126. Kang, D.H., et al., The effect of meditation on brain structure: cortical thickness mapping and diffusion tensor imaging. Soc Cogn Affect Neurosci, 2012.

127. Slagter, H.A., R.J. Davidson, and A. Lutz, Mental training as a tool in the neuroscientific study of brain and cognitive plasticity. Front Hum Neurosci, 2011. 5: p. 17.

128. Lazar, S.W., et al., Meditation experience is associated with increased cortical thickness. Neuroreport, 2005. 16(17): p. 1893-7.

129. Gard, T., B.K. Holzel, and S.W. Lazar, The potential effects of meditation on age-related cognitive decline: a systematic review. Ann N Y Acad Sci, 2014. 1307: p. 89-103.

130. Shonin, E., et al., Buddhist-derived loving-kindness and compassion meditation for the treatment of psychopathology: a systematic review. Mindfulness, 2015. 6(5): p. 1161-1180.

131. Pattanashetty, R., et al., Practitioners of vipassana meditation exhibit enhanced slow wave sleep and REM sleep states across different age groups. Sleep and Biological Rhythms, 2009. 8(1): p. 34-41.

132. Cote, K.A., T.M. Epps, and K.B. Campbell, The role of the spindle in human information processing of high-intensity stimuli during sleep. J Sleep Res, 2000. 9(1): p. 19-26.

133. Dang-Vu, T.T., et al., Interplay between spontaneous and induced brain activity during human nonrapid eye movement sleep. Proc Natl Acad Sci U S A, 2011. 108(37): p. 15438-43.

134. Dang-Vu, T.T., et al., Spontaneous brain rhythms predict sleep stability in the face of noise. Curr Biol, 2010. 20(15): p. R626-7.

135. Lecci, S., et al., Coordinated infraslow neural and cardiac oscillations mark fragility and offline periods in mammalian sleep. Sci Adv, 2017. 3(2): p. e1602026.

136. Dang-Vu, T.T., et al., Sleep spindles predict stress-related increases in sleep disturbances. Front Hum Neurosci, 2015. 9: p. 68. 
137. Mezick, E.J., et al., Intra-individual variability in sleep duration and fragmentation: associations with stress. Psychoneuroendocrinology, 2009. 34(9): p. 1346-54.

138. MacLean, K.A., et al., Intensive meditation training improves perceptual discrimination and sustained attention. Psychol Sci, 2010. 21(6): p. 829-39.

139. Britton, W.B., et al., Awakening is not a metaphor: the effects of Buddhist meditation practices on basic wakefulness. Ann N Y Acad Sci, 2014. 1307: p. 64-81.

140. Engel, A.K., P. Fries, and W. Singer, Dynamic predictions: oscillations and synchrony in top-down processing. Nat Rev Neurosci, 2001. 2(10): p. 704-16.

141. Steinmetz, P.N., et al., Attention modulates synchronized neuronal firing in primate somatosensory cortex. Nature, 2000. 404(6774): p. 187-90.

142. Lutz, A., et al., Long-term meditators self-induce high-amplitude gamma synchrony during mental practice. Proc Natl Acad Sci U S A, 2004. 101(46): p. 16369-73.

143. Lachaux, J.P., et al., Measuring phase synchrony in brain signals. Hum Brain Mapp, 1999. 8(4): p. 194-208.

144. Varela, F., et al., The brainweb: phase synchronization and large-scale integration. Nat Rev Neurosci, 2001. 2(4): p. 229-39.

145. Uhlhaas, P.J. and W. Singer, Neural synchrony in brain disorders: relevance for cognitive dysfunctions and pathophysiology. Neuron, 2006. 52(1): p. 155-68.

146. Steriade, M. and I. Timofeev, Neuronal plasticity in thalamocortical networks during sleep and waking oscillations. Neuron, 2003. 37(4): p. 563-76.

147. Zerouali, Y., et al., A time-frequency analysis of the dynamics of cortical networks of sleep spindles from MEG-EEG recordings. Front Neurosci, 2014. 8: p. 310.

148. Eichenlaub, J.B., et al., Brain reactivity differentiates subjects with high and low dream recall frequencies during both sleep and wakefulness. Cereb Cortex, 2014. 24(5): p. 1206-15.

149. Eichenlaub, J.B., et al., Resting brain activity varies with dream recall frequency between subjects. Neuropsychopharmacology, 2014. 39(7): p. 1594-602.

150. Fogel, S.M. and C.T. Smith, The function of the sleep spindle: a physiological index of intelligence and a mechanism for sleep-dependent memory consolidation. Neurosci Biobehav Rev, 2011. 35(5): p. 1154-65.

151. Ujma, P.P., et al., Sleep spindles and intelligence in early childhood-developmental and traitdependent aspects. Dev Psychol, 2016. 52(12): p. 2118-2129.

152. Bodizs, R., et al., Sleep spindling and fluid intelligence across adolescent development: sex matters. Front Hum Neurosci, 2014. 8(952).

153. Ujma, P.P., et al., Sleep spindles and intelligence: evidence for a sexual dimorphism. J Neurosci, 2014. 34(49): p. 16358-68.

154. Kaestner, E.J., J.T. Wixted, and S.C. Mednick, Pharmacologically Increasing Sleep Spindles Enhances Recognition for Negative and High-arousal Memories. J Cogn Neurosci, 2013.

155. Gard, T., et al., Fluid intelligence and brain functional organization in aging yoga and meditation practitioners. Front Aging Neurosci, 2014. 6: p. 76. 
156. Chambers, R., E. Gullone, and N.B. Allen, Mindful emotion regulation: An integrative review. Clin Psychol Rev, 2009. 29(6): p. 560-72.

157. Brokaw, K., et al., Resting state EEG correlates of memory consolidation. Neurobiol Learn Mem, 2016. 130: p. 17-25. 

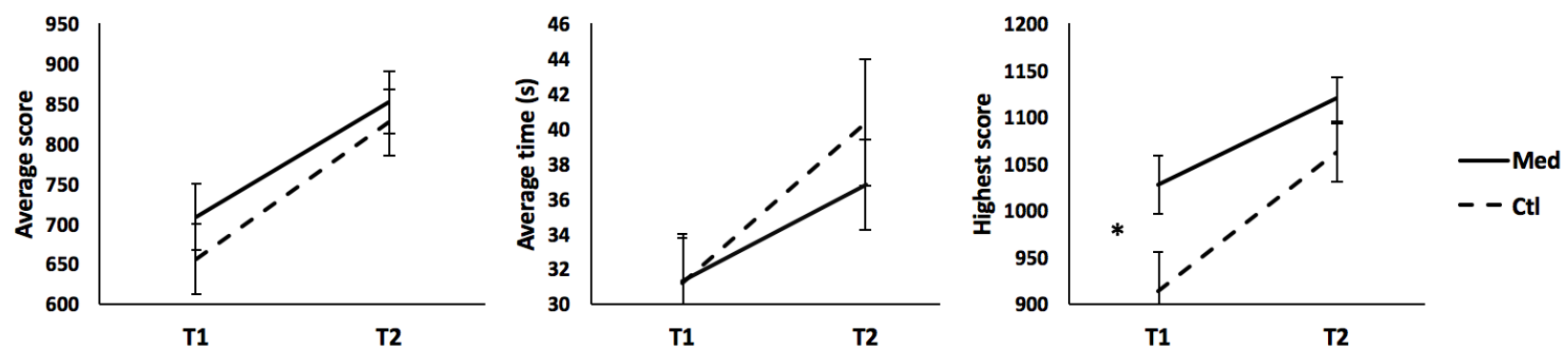

Figure 1. Performance on procedural balance task in meditators (MED) and non-meditating controls (CTL) before nap (T1) and after nap (T2). ${ }^{*} \mathrm{p}<.05$ 
Table 1. Paired samples t-tests: comparison of task scores before and after the nap in meditators (MED) and non-meditating controls (CTL).

\begin{tabular}{|c|c|c|c|c|c|}
\hline & \multicolumn{5}{|c|}{ MED } \\
\hline & \multirow{2}{*}{$\begin{array}{l}\text { Mean } \pm \text { SD } \\
\text { Before nap }\end{array}$} & \multirow{2}{*}{$\begin{array}{c}\text { Mean } \pm \text { SD } \\
\text { After nap }\end{array}$} & \multicolumn{2}{|c|}{$\begin{array}{c}\text { Paired sample } \\
\text { correlations }\end{array}$} & \multirow{2}{*}{$\begin{array}{c}\text { Paired t-test } \\
p\end{array}$} \\
\hline & & & $\mathbf{R}$ & $p$ & \\
\hline Time & $31.31 \pm 10.98$ & $36.85 \pm 11.35$ & 0.71 & 0.000 & 0.009 \\
\hline GameScore & $\begin{array}{l}708.43 \pm \\
184.71\end{array}$ & $852.35 \pm 170.19$ & 0.75 & 0.000 & 0.000 \\
\hline \multirow[t]{4}{*}{ HiScore } & $\begin{array}{c}1028.40 \pm \\
142.21\end{array}$ & $1120.15 \pm 106.44$ & 0.48 & 0.034 & 0.006 \\
\hline & \multicolumn{5}{|c|}{ CTL } \\
\hline & Mean \pm SEM & Mean \pm SEM & \multicolumn{2}{|c|}{$\begin{array}{l}\text { Paired sample } \\
\text { correlations }\end{array}$} & Paired t-test \\
\hline & Before nap & After nap & $\mathbf{R}$ & $p$ & $p$ \\
\hline Time & $31.20 \pm 12.66$ & $40.38 \pm 16.11$ & 0.68 & 0.001 & 0.003 \\
\hline GameScore & $\begin{array}{l}656.87 \pm \\
199.14\end{array}$ & $827.98 \pm 186.02$ & 0.78 & 0.000 & 0.000 \\
\hline HiScore & $913.25 \pm 187.54$ & $1061.95 \pm 141.42$ & 0.63 & 0.003 & 0.000 \\
\hline
\end{tabular}


MED before nap

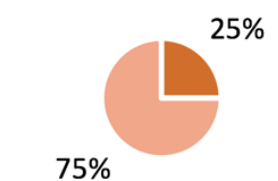

- Finished $=$ Unfinished

MED after nap

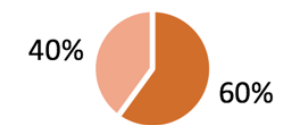

- Finished $=$ Unfinished

\section{CTL before nap}

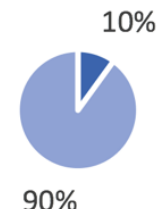

$90 \%$

- Finished $=$ Unfinished

CTL after nap

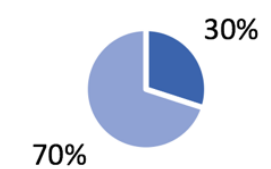

- Finished $=$ Unfinished

Figure 2. Proportion of meditators (MED) and controls (CTL) who did (Finished) and did not (Unfinished) complete the procedural task at least once before and after the daytime nap 
Table 2. Scores on procedural task for Vipassana meditators (MED) and non-meditating controls (CTL). ${ }^{*} \mathrm{p}<.05 ; \uparrow \mathrm{p}$ $<.10$

\begin{tabular}{lccccc}
\hline Task variable & Mean MED \pm SD & Mean CTL \pm SD & t & df & p \\
\hline T1 HiScore** & $1028.40 \pm 142.21$ & $913.25 \pm 187.54$ & 2.19 & 38.00 & 0.04 \\
T1 Time & $31.31 \pm 10.97$ & $31.20 \pm 12.66$ & 0.03 & 38.00 & 0.98 \\
T1 GameScore & $708.43 \pm 184.71$ & $656.87 \pm 199.16$ & 0.85 & 38.00 & 0.40 \\
T1 \#Wins & $0.30 \pm 0.57$ & $0.10 \pm 0.31$ & 1.38 & 29.18 & 0.18 \\
T2 HiScore & $1120.15 \pm 106.44$ & $1061.95 \pm 141.42$ & 1.47 & 35.30 & 0.15 \\
T2 Time & $36.85 \pm 11.35$ & $40.38 \pm 16.11$ & - & 38.00 & 0.43 \\
& & & 0.80 & & \\
T2 GameScore & $852.35 \pm 170.19$ & $827.98 \pm 186.02$ & 0.43 & 38.00 & 0.67 \\
T2 TotScore & $7322.75 \pm 1631.78$ & $6458.45 \pm 1806.54$ & 1.59 & 38.00 & 0.12 \\
T2 \#Wins* & $0.95 \pm 0.10$ & $0.45 \pm 0.83$ & 1.73 & 38.00 & 0.09 \\
T2-T1Time & $5.54 \pm 8.46$ & $9.18 \pm 11.97$ & - & 38.00 & 0.28 \\
T2-T1Score & $143.93 \pm 126.76$ & $171.12 \pm 129.26$ & - & 38.00 & 0.51 \\
& & & 0.67 & & \\
\hline
\end{tabular}

$T 1$ refers to procedural task completed before the daytime nap; T2 refers to the same task completed after the nap. Score was calculated in "meters" according to the gameplay distance measure. Time was measured in seconds. Game completion was measured as a number of times participants arrived at the end of the virtual river. Improvement on task was measured as the difference between post-nap and pre-nap performance (T2-T1). 

$<.10$

Table 3. Sleep measures for Vipassana meditators (MED: N=20) and non-meditating controls (CTL: $N=20)$. $\uparrow p$

\begin{tabular}{lccrrr}
\hline Sleep characteristic & Mean MED \pm SD & Mean CTL \pm SD & \multicolumn{1}{c}{ t } & df & p \\
\hline Total sleep duration (min) & $65.98 \pm 25.27$ & $78.30 \pm 19.32$ & -1.73 & 38.00 & $0.09 \dagger$ \\
Sleep latency (min) & $8.78 \pm 6.75$ & $14.35 \pm 20.08$ & 0.20 & 38.00 & 0.84 \\
REM latency (min) & $16.35 \pm 22.35$ & $20.74 \pm 31.23$ & -0.47 & 32.00 & 0.64 \\
Sleep efficiency & $73.66 \pm 23.10$ & $81.70 \pm 13.31$ & -1.31 & 29.68 & 0.20 \\
N1 duration (min) & $7.13 \pm 7.67$ & $7.03 \pm 3.81$ & 0.05 & 38.00 & 0.96 \\
N2 duration (min) & $32.83 \pm 16.47$ & $36.05 \pm 15.05$ & -0.65 & 38.00 & 0.52 \\
N3 duration (min) & $15.05 \pm 13.38$ & $22.53 \pm 18.99$ & -1.44 & 38.00 & 0.16 \\
Total NREM duration (min) & $55.00 \pm 23.28$ & $65.60 \pm 22.37$ & -1.47 & 38.00 & 0.15 \\
REM duration (min) & $10.98 \pm 6.12$ & $12.70 \pm 9.99$ & -0.66 & 38.00 & 0.51 \\
Wake duration (min) & $24.75 \pm 24.71$ & $17.45 \pm 12.33$ & 1.18 & 27.91 & 0.24 \\
N1\% & $13.31 \pm 16.30$ & $10.45 \pm 8.94$ & 0.69 & 38.00 & 0.50 \\
N2\% & $50.55 \pm 17.18$ & $45.10 \pm 15.68$ & -0.90 & 38.00 & 0.50 \\
N3\% & $19.46 \pm 15.95$ & $26.45 \pm 20.43$ & -1.21 & 38.00 & 0.24 \\
NREM\% & $83.32 \pm 10.68$ & $82.90 \pm 15.17$ & 0.10 & 38.00 & 0.92 \\
REM\% & $16.69 \pm 10.68$ & $17.11 \pm 15.17$ & -0.10 & 38.00 & 0.92 \\
Wake\% & $26.29 \pm 24.02$ & $18.30 \pm 13.31$ & 1.30 & 29.66 & 0.20 \\
N awakenings & $5.65 \pm 3.84$ & $6.70 \pm 4.18$ & -0.83 & 38.00 & 0.41 \\
\hline
\end{tabular}




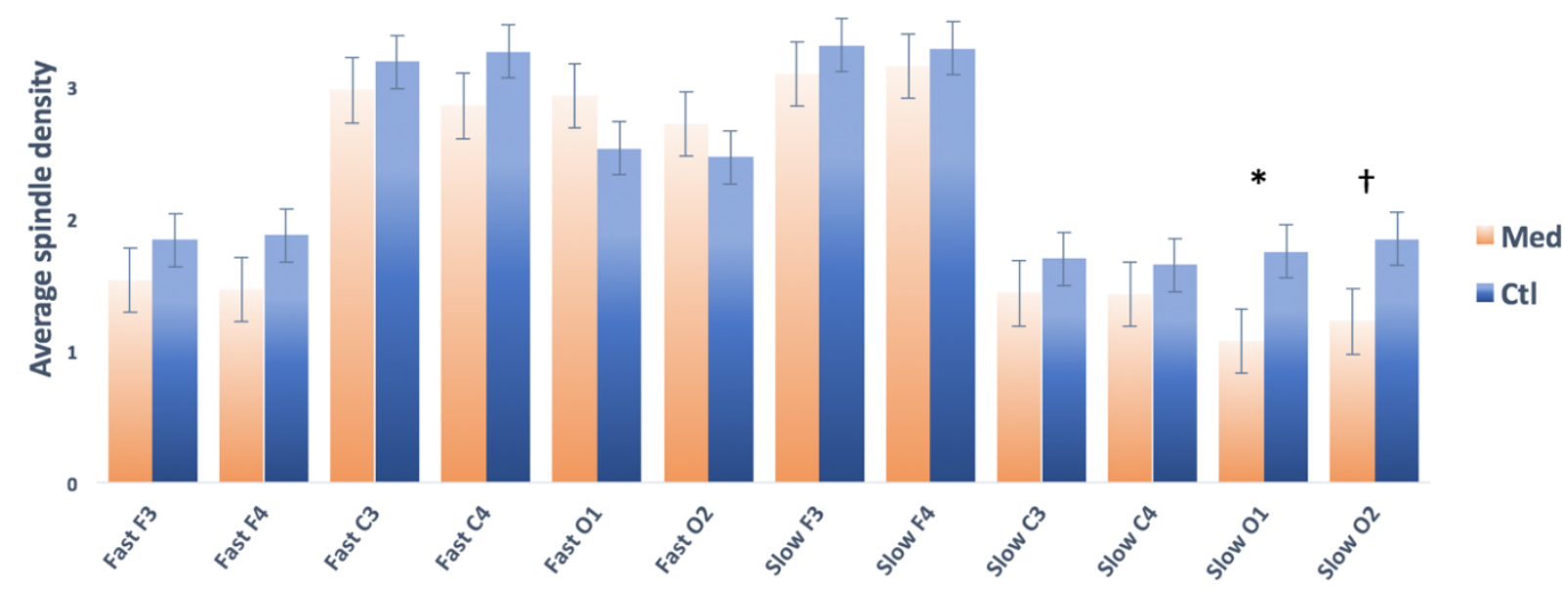

Figure 3. Sleep spindle densities ( $\mathrm{M} \pm \mathrm{SEM}$ ) for Vipassana meditators (MED; $\mathrm{N}=20$ ) and non-meditating controls (CTL; $\mathrm{N}=20$ ). Spindle density calculated as the number of sleep spindles/time in NREM2 sleep. ${ }^{*} \mathrm{p}<.05 ; \mathrm{p}<.06$ 
Balance task improvement: time

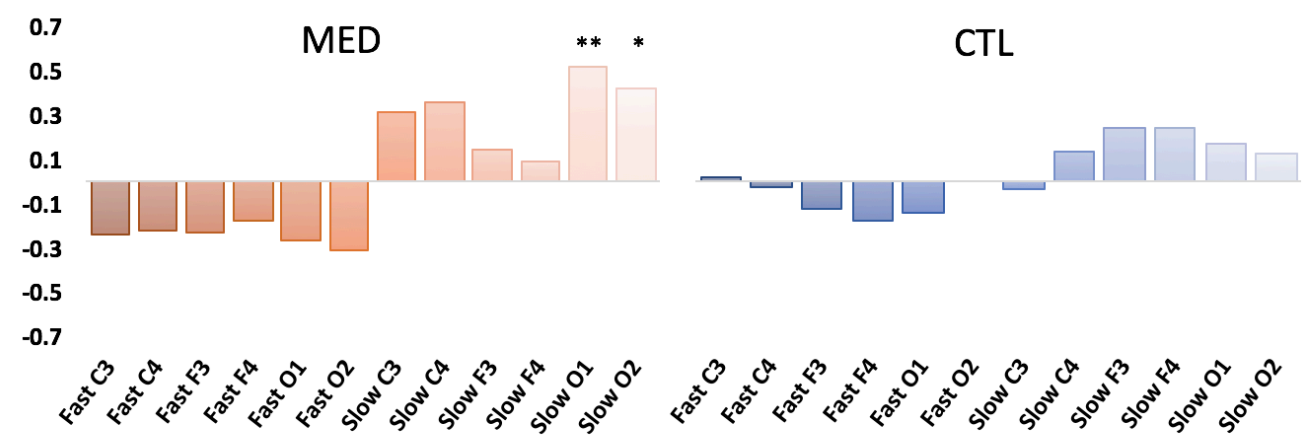

\section{Balance task improvement: score}

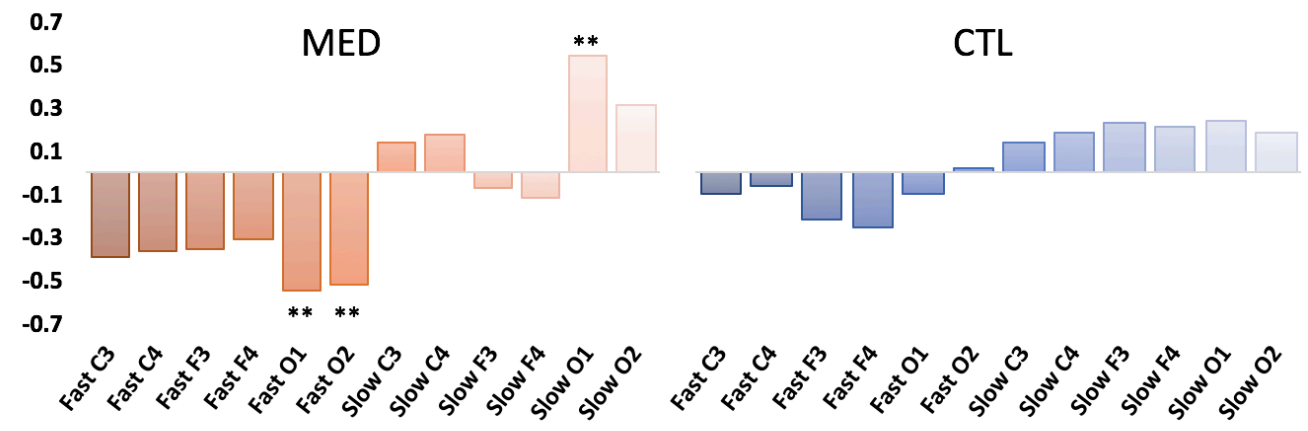

Figure 4. Pearson correlations between fast and slow sleep spindle densities and post-nap improvement on the balance task (average time and score) in meditators (MED) and controls (CTL). ${ }^{*} \mathrm{p}<.05 ; * * \mathrm{p}<.01$ 
Table 4. Pearson correlation coefficients between sleep characteristics and post-nap improvement in performance on a procedural task in meditators (MED) and controls (CTL). Average improvement in time and in score is reported. $*=\mathrm{p}<.05 ; * *=\mathrm{p}<.01 ; * * *=\mathrm{p}<.001$ (Conservative error rate adjustment for 32 correlations per group $=32 / .05=.002$ ).

\begin{tabular}{lrrrrrr}
\hline & \multicolumn{3}{c}{ MED } & \multicolumn{3}{c}{ CTL } \\
\hline Sleep variable & Time & Score & Time & Score \\
\hline total sleep duration & 0.31 & 0.06 & -0.10 & & 0.01 & \\
sleep latency & 0.16 & 0.20 & -0.20 & -0.48 & $* *$ \\
REM latency & -0.02 & 0.18 & 0.28 & & 0.26 & \\
Sleep efficiency & 0.20 & 0.04 & 0.29 & & 0.45 & $* *$ \\
N1 duration & 0.01 & -0.09 & -0.47 & $* *$ & -0.47 & $* *$ \\
N2 duration & 0.22 & 0.14 & -0.39 & $*$ & -0.23 & \\
N3 duration* & 0.20 & -0.01 & -0.01 & & 0.01 & \\
Total NREM & 0.30 & 0.06 & -0.35 & & -0.23 & \\
duration* & & & & & & \\
REM duration & 0.11 & 0.00 & 0.59 & $* * *$ & 0.54 & $* *$ \\
Wake duration & -0.15 & -0.02 & -0.32 & & -0.50 & $* *$ \\
N1\% & -0.11 & -0.14 & -0.41 & $*$ & -0.42 & $*$ \\
N2\% & 0.02 & 0.20 & -0.42 & $*$ & -0.33 & \\
N3\% & 0.12 & -0.07 & -0.01 & & 0.00 & \\
NREM\% & 0.05 & -0.01 & -0.66 & $* * *$ & -0.57 & $* * *$ \\
REM\% & -0.05 & 0.01 & 0.66 & $* * *$ & 0.57 & $* * *$ \\
Wake\% & -0.20 & -0.04 & -0.29 & & -0.45 & $* *$
\end{tabular}

